\title{
Bergamottin contribution to the grapefruit juice-felodipine interaction and disposition in humans
}

Objectives: Our objectives were to evaluate the contribution of bergamottin to the grapefruit juice-felodipine interaction and to characterize bergamottin disposition.

Methods: In this study $250 \mathrm{~mL}$ grapefruit juice; 2-, 6-, or 12-mg capsules of bergamottin plus water; or water was administered with $5 \mathrm{mg}$ extended-release felodipine to 11 volunteers in a partially randomized, 5-way crossover study. Plasma concentrations of felodipine, its primary metabolite (dehydrofelodipine), bergamottin, and $6^{\prime}, 7^{\prime}$-dihydroxybergamottin were determined.

Results: Grapefruit juice (containing $1.7 \mathrm{mg}$ bergamottin) increased peak plasma concentration $\left(\mathrm{C}_{\max }\right)$ and area under the plasma concentration-time curve (AUC) of felodipine by $89 \%(P<.025)$ and $54 \%(P<.025)$, respectively, compared with water. With $2 \mathrm{mg}$ bergamottin, felodipine $\mathrm{C}_{\max }$ increased by $33 \%(P<.05)$. The increase by bergamottin was markedly variable among individuals (range, $-33 \%$ to $125 \%$ ). With $6 \mathrm{mg}$ bergamottin, felodipine $\mathrm{C}_{\max }$ was enhanced by $35 \%(P<.025)$, and with $12 \mathrm{mg}$ bergamottin, felodipine $\mathrm{C}_{\max }$ increased by $40 \%(P<.05)$ and AUC increased by $37 \%(P<.05)$ compared with water. Bergamottin measured in plasma after administration of 6 and $12 \mathrm{mg}$ produced $C_{\max }$ values of 2.1 and $5.9 \mathrm{ng} / \mathrm{mL}$, respectively, and times to reach $C_{\max }$ of 0.8 and 1.1 hours, respectively. The bergamottin metabolite $6^{\prime}, 7^{\prime}$-dihydroxybergamottin was detected in plasma of some subjects after bergamottin administration.

Conclusions: Bergamottin enhanced the oral bioavailability of felodipine and may cause a clinically relevant drug interaction in susceptible individuals. Grapefruit juice-drug interactions likely also involve other furanocoumarins, possibly acting in combination by additive or synergistic mechanisms. Bergamottin has systemic availability and is metabolized in vivo to $6^{\prime}, 7^{\prime}$-dihydroxybergamottin. (Clin Pharmacol Ther 2004; 76:607-17.)

Theunis C. Goosen, PhD, Doré Cillié, MSc, David G. Bailey, PhD, Chongwoo Yu, PhD, Kan He, PhD, Paul F. Hollenberg, PhD, Patrick M. Woster, PhD, Lucinda Cohen, PhD, J. Andrew Williams, PhD, Malie Rheeders, PhD, and H. Paul Dijkstra, MD Potchefstroom, South Africa, London, Ontario, Canada, and Ann Arbor and Detroit, Mich

Grapefruit juice was initially shown to increase the oral bioavailability of medications from a wide range of therapeutic categories, including the antihypertensive dihydropyridine calcium antagonist felodipine. ${ }^{1}$ Felo-

From the Department of Pharmacology, North-West University, Potchefstroom; Department of Medicine, London Health Sciences Centre, University of Western Ontario, London; Department of Pharmacokinetics, Dynamics, and Metabolism, Pfizer Global Research and Development, and Department of Pharmacology, University of Michigan, Ann Arbor; and Department of Pharmaceutical Sciences, Wayne State University, Detroit.

Supported in part by grants from the Medical Research Council of South Africa (02M03) and Canadian Institute of Health Research (MOP 67013).

Presented in part at the Fifty-second Annual Meeting of the American Society for Mass Spectrometry, Nashville, Tenn, May 23-27, 2004. dipine is normally completely absorbed from the gastrointestinal tract after oral administration but has a low absolute bioavailability (15\%) as a result of extensive presystemic metabolism mediated by cytochrome $\mathrm{P} 450$ (CYP) 3A4. ${ }^{2}$ Grapefruit juice coadministration resulted in a several-fold increase in peak plasma concentration

Received for publication June 7, 2004; accepted Aug 25, 2004. Reprint requests: Theunis C. Goosen, Department of Pharmacokinetics, Dynamics, and Metabolism, Pfizer Global Research and Development, Bldg 20-322, 2800 Plymouth Rd, Ann Arbor, MI 48105.

E-mail: theunis.goosen@pfizer.com

0009-9236/\$30.00

Copyright () 2004 by the American Society for Clinical Pharmacology and Therapeutics.

doi:10.1016/j.clpt.2004.08.019 
<smiles>CC(C)=CCC/C(C)=C/COc1c2ccoc2cc2oc(=O)ccc12</smiles>

\section{Bergamottin}<smiles>CC(=CCOc1c2ccoc2cc2oc(=O)ccc12)CCC(O)C(C)(C)O</smiles>

\section{6',7'-Dihydroxybergamottin}

Fig 1. Chemical structures of bergamottin and $6^{\prime}, 7^{\prime}-$ dihydroxybergamottin.

$\left(\mathrm{C}_{\max }\right)$ and the area under the plasma concentrationtime curve (AUC) of felodipine and its primary metabolite, dehydrofelodipine, and a decrease in the dehydrofelodipine/felodipine AUC ratio. ${ }^{3,4}$ This was attributed to inhibition of both the primary and secondary steps in the metabolism of felodipine. Grapefruit juice at normal volume did not change the terminal half-life $\left(t_{1 / 2}\right)$ or intravenous pharmacokinetics of drugs. ${ }^{1,5-7}$ Therefore this pharmacokinetic interaction is thought to be due primarily to grapefruit juicemediated inhibition of intestinal CYP3A4 activity without apparent inhibition of hepatic CYP3A4 activity. Grapefruit juice inhibition of CYP3A4 in vivo appears to involve irreversible inactivation of CYP3A4, as evidenced by down-regulation of intestinal CYP3A4 protein content without alteration of intestinal messenger ribonucleic acid levels. ${ }^{3,8}$ This posttranslational mechanism most likely resulted from mechanism-based inactivation of intestinal CYP3A4, ultimately resulting in protein degradation. ${ }^{9}$ Evidence further suggests that grapefruit juice might also inhibit P-glycoprotein, although clinical support is still controversial. ${ }^{10-15}$ The grapefruit juice interaction has subsequently been shown to be relevant for a number of medications including terfenadine, cisapride, halofantrine, lovastatin, simvastatin, amiodarone, buspirone, tacrolimus, and cyclosporine (INN, ciclosporin). ${ }^{3,16}$

Initial studies investigating the effect of flavonoids as in vivo CYP3A4 inhibitors failed to show significant activity in humans. ${ }^{17-19}$ More recent efforts have fo- cused on furanocoumarins. Bergamottin (Fig 1) is present in grapefruit juice in concentrations ranging from 2 to $30 \mu \mathrm{mol} / \mathrm{L},{ }^{12,20,21}$ depending on the source of the juice. These concentrations approach or exceed those for half-maximal enzyme inactivation $\left(\mathrm{K}_{\mathrm{I}}\right)$ determined by use of expressed CYP3A4 $(7.7 \mu \mathrm{mol} / \mathrm{L})^{22}$ or human liver microsomes $(40 \mu \mathrm{mol} / \mathrm{L}){ }^{23}$ The $50 \%$ inhibitory concentration for nifedipine oxidation determined with recombinant protein $(5.4 \mu \mathrm{mol} / \mathrm{L})^{24}$ is also lower than in human liver microsomes $(22 \mu \mathrm{mol} / \mathrm{L}$, testosterone $6 \beta$-hydroxylation). ${ }^{21}$ The more hydrophilic metabolite, $6^{\prime}, 7^{\prime}$-dihydroxybergamottin, is often present in grapefruit juice in similar concentrations $(0.8$ to $58 \mu \mathrm{mol} / \mathrm{L}),{ }^{21,25}$ but initial investigations indicated that it is a less potent in vitro mechanism-based inactivator of CYP3A4 $\left(\mathrm{K}_{\mathrm{I}}, 59 \mu \mathrm{mol} / \mathrm{L}\right)$ than bergamottin. ${ }^{25}$ However, recent reports by different groups indicate that $6^{\prime}, 7^{\prime}$-dihydroxybergamottin is a more potent mechanism-based inactivator or inhibitor of CYP3A4. ${ }^{23,26}$ Interestingly, 50\% inhibitory concentration values determined for inhibition of CYP3A4 activity vary by 2 orders of magnitude and range between 0.3 to $26 \mu \mathrm{mol} / \mathrm{L}^{24-28}$ Clinically, $6^{\prime}, 7^{\prime}$-dihydroxybergamottin administered as the aqueous fraction of grapefruit juice increased the oral bioavailability of felodipine. The magnitude of interaction indicated that $6^{\prime}, 7^{\prime}$-dihydroxybergamottin was unlikely to be the primary active constituent. ${ }^{29}$ However, a recent study in a smaller population suggested a larger contribution of $6^{\prime}, 7^{\prime}$-dihydroxybergamottin than previously reported. ${ }^{30}$

Bergamottin is both a competitive inhibitor and a mechanism-based inactivator of CYP3A4 in human liver microsomes and recombinant complementary deoxyribonucleic acid-expressed protein. ${ }^{21-23}$ In addition, bergamottin has been shown to be a substrate inhibitor of P-glycoprotein in whole cell-based assays. ${ }^{31}$ Because mechanism-based inactivation can result in protein degradation in vivo and bergamottin is one of the major furanocoumarins in the juice, bergamottin might be a principal constituent responsible for the interaction in humans. ${ }^{22,32}$ Administration of a particulate portion of grapefruit juice (containing high concentrations of bergamottin) enhanced bioavailability of felodipine more than the supernatant portion (containing no detectable bergamottin and high concentrations of $6^{\prime}, 7^{\prime}$-dihydroxybergamottin). ${ }^{29}$ However, dilute lime juice containing the same concentration of bergamottin produced much less interaction than did whole grapefruit juice. ${ }^{33}$ Bergamottin coadministration increased the bioavailability of diazepam in beagle dogs by 54-fold and the bioavailability of cyclosporine and nifedipine in rats by 3.4-fold and 1.5-fold, 
respectively. ${ }^{34-36}$ However, relative exposure to bergamottin is not known, and doses administered were typically larger than those encountered by humans after normal consumption of grapefruit juice. Thus the relevance of bergamottin in the clinical interaction of grapefruit juice in humans is currently uncertain.

Determining the role of bergamottin might ideally involve administering this furanocoumarin as a pure substance. The purpose of this study was to assess the effect of 3 escalating doses of pure bergamottin, administered as an ethanolic solution in gelatin capsules, on the oral pharmacokinetics of felodipine in humans. Results showed that bergamottin at a dose similar to that in grapefruit juice augmented the oral bioavailability of felodipine but the interaction was much less than that produced by the juice.

\section{METHODS}

\section{Materials}

Grapefruit juice concentrate (Cedar Lite; Bromor Foods, Cape Town, South Africa) was obtained from a local market. Bergamottin was purchased from Indofine Chemical Co (Somerville, NJ) and was of the highest purity $(100 \%)$ commercially available. Purity was confirmed by HPLC $(>99.8 \%)$ with ultraviolet (UV) detection $(310 \mathrm{~nm})^{21}$ and liquid chromatography-tandem mass spectrometry (LC-MS/MS) analyses essentially as later described. Trioxsalen (INN, trioxysalen) and bergapten were purchased from Sigma-Aldrich (St Louis, Mo), and 6',7'-dihydroxybergamottin was supplied by Ultrafine Chemicals (Manchester, England). All other materials were reagent-grade and obtained from commercial sources.

\section{Analysis of bergamottin concentration in grapefruit juice}

Bergamottin concentration in grapefruit juice concentrate (Cedar Lite; Bromor Foods) was analyzed essentially according to a previously published method. ${ }^{15}$ Grapefruit juice was diluted with water, and an aliquot $(1 \mathrm{~mL})$ was extracted twice with an equal volume of ethyl acetate and once with dichloromethane by shaking for 45 minutes. Extracts were combined after centrifugation, dried under a gentle stream of nitrogen in an ice bath, and dissolved in $200 \mu \mathrm{L}$ mobile phase (65\% acetonitrile) containing $100 \mathrm{nmol}$ of trioxsalen as internal standard. The grapefruit juice components were separated by HPLC (Agilent Hewlett-Packard 1100; Chemetrix Ltd, Midrand, South Africa) equipped with a diode array detector. An aliquot $(20 \mu \mathrm{L})$ was injected onto a C18 column (Luna, $5 \mu \mathrm{m}, 4.6 \times 250$ $\mathrm{mm}$; Phenomenex, Torrance, Calif) at a flow rate of 1
$\mathrm{mL} / \mathrm{min}$, and elution was performed with $100-\mathrm{mmol} / \mathrm{L}$ acetic acid (A) and acetonitrile (B) by a gradient of $40 \%$ B for 5 minutes and then $40 \%$ to $70 \%$ B within 25 minutes and held for 5 minutes before returning to initial conditions. UV absorbance was measured at 310 $\mathrm{nm}$ and quantified according to a standard curve generated between 1 and $100 \mu \mathrm{mol} / \mathrm{L}$. The standard curve was linear over the range tested $\left(r^{2}>0.999\right)$ and had a coefficient of variation of $4.2 \%$ at $1 \mu \mathrm{mol} / \mathrm{L}(\mathrm{n}=3)$. The lower limit of quantitation for bergamottin was 0.5 $\mu \mathrm{mol} / \mathrm{L}$, and the interday coefficient of variation was $5.9 \%$ at $20 \mu \mathrm{mol} / \mathrm{L}$. Bergamottin and trioxsalen eluted at 31.9 and 14.2 minutes, respectively, and bergamottin recovery from spiked orange juice (not containing bergamottin) was $97 \% \pm 6 \%(\mathrm{n}=3)$. The coefficient of variation for extracted bergamottin was $8.2 \%$ at 20 $\mu \mathrm{mol} / \mathrm{L}$. Analysis for assay specificity was performed by coinjection and indicated no split peaks. In addition, the UV absorption profile (200 to $350 \mathrm{~nm}$ ) of the assayed chromatographic peak from the ethyl acetate/ dichloromethane extract was identical to that of pure bergamottin. Concentrations of other furanocoumarins in the juice were not quantified because of the lack of commercially available standards at the time of analysis.

\section{Human pharmacokinetic study}

Subjects. Twelve white male volunteers (age range, 21-24 years) gave written informed consent before entering the study as approved by the Ethics Committee for Human Research of North-West University (Potchefstroom, South Africa) (02M03). As a result of the limited availability of Good Manufacturing Practice bergamottin and individual regulations on food substances, future studies may not be permitted in some countries. The clinical study was performed at the Drug Bioavailability Centrum, Department of Pharmacology, North-West University, and was conducted in accordance with the Declaration of Helsinki. The subjects were ascertained to be healthy by medical history, physical examination, routine hematologic testing, and blood and urine chemistry studies. All subjects had a normal 12-lead electrocardiogram and tested negative for human immunodeficiency virus 1 or 2 antibody, hepatitis B antigen, or hepatitis $\mathrm{C}$ antibody at screening. None of the subjects was receiving any medication on a long-term basis, and all were purported to be nonsmokers.

Bergamottin capsules. Bergamottin dosing solutions were prepared in absolute ethanol to yield stock solutions of 4,12 , or $24 \mathrm{mg} / \mathrm{mL}$ directly before administration on each experimental day. Soft gelatin cap- 
sules were filled with $500 \mu \mathrm{L}$ of the respective stock solutions for oral administration of 2,6 , or $12 \mathrm{mg}$ bergamottin. Bergamottin was used without further physicochemical characterization. However, the purity of bergamottin in dosing solutions was confirmed by liquid chromatography-diode array detector analyses by use of an HPLC system equipped with a diode array detector for UV detection, as well as subsequent LCMS/MS analyses by use of the same chromatographic separation conditions described for the analysis of bergamottin in grapefruit juice. UV spectra were recorded over the range of 200 to $360 \mathrm{~nm}$. As a result, only 1 peak was detected in our liquid chromatography-diode array detector analysis $\left(>99.8 \%\right.$ at $\lambda_{\max }=310 \mathrm{~nm}$, where $\lambda_{\max }$ is wavelength of maximum absorption) that was confirmed to be pure bergamottin on the basis of the subsequent mass spectrometric analysis results showing characteristic fingerprints of bergamottin. Mass spectra were recorded over the range of mass-tocharge ratio $(\mathrm{m} / \mathrm{z}) 100$ to 1000 for full scan analysis, and protonated molecule of bergamottin $\left([\mathrm{M}+\mathrm{H}]^{+}=\right.$ $\mathrm{m} / \mathrm{z}$ 339) was detected as the base peak. Characteristic product ions of $\mathrm{m} / \mathrm{z}, 203,175$, and 137 were detected in product ion analysis that were consistent with previous findings. ${ }^{22}$ No other peaks or ions possibly related to the synthesis materials (bergaptol or geranyl bromide) were detected.

Protocol. Subjects received racemic felodipine in a 5-mg extended-release formulation (Plendil; AstraZeneca, Sunninghill, South Africa) with (1) $250 \mathrm{~mL}$ grapefruit juice (Cedar Lite; Bromor Foods) as positive control or (2) water (negative control) in a randomized, 2-way crossover manner followed by $250 \mathrm{~mL}$ water with (3) $2 \mathrm{mg}$, (4) $6 \mathrm{mg}$, or (5) $12 \mathrm{mg}$ bergamottin (gelatin capsule containing $500 \mu \mathrm{L}$ ethanol) in a singledose, randomized, 3-way crossover manner. The 2-mg, 6-mg, or 12-mg bergamottin dose administered would be equivalent to that encountered after consumption of approximately $1,3.5$, or 7 glasses $(250 \mathrm{~mL})$ of Cedar Lite grapefruit juice, respectively. The same lot number of grapefruit juice was used throughout the study. Peripheral venous blood $(10 \mathrm{~mL})$ was sampled just before dosing and at $0.5,1,1.5,2,2.5,3,4,5,6,8,10,12$, and 24 hours after dosing for the purpose of measuring plasma felodipine, dehydrofelodipine, bergamottin, or $6^{\prime}, 7^{\prime}$-dihydroxybergamottin concentrations. Plasma samples were split for felodipine and bergamottin analyses and stored at $-80^{\circ} \mathrm{C}$ after immediate centrifugation. Plasma was shipped under dry ice, and samples were stored at $-80^{\circ} \mathrm{C}$ before analyses as described below. Subjects consumed standardized meals at 5 hours after dosing (1 PM) and 8 hours after dosing (4
PM). Consumption of beverages that contained caffeine was not allowed during testing, and water was allowed starting at 3 hours after dosing. Subjects were not permitted to consume alcohol for at least 48 hours and fasted for at least 10 hours before testing. They were instructed not to consume grapefruit juice products, other than the one used in the study, for 2 weeks before and during the period of investigation. Compliance to this criterion was determined by measuring predose plasma bergamottin concentrations in all subjects before each treatment phase. Subjects were prohibited from taking prescription drugs, over-the-counter medications, and herbal supplements within 30 days before and during the study. The washout interval between study days was 1 week. Patients were under the constant supervision of a licensed physician and registered nurse for the duration of the study. Adverse events were recorded by questionnaire, and volunteers were encouraged to immediately report any change in well-being.

Plasma analysis for felodipine and dehydrofelodipine. Plasma concentrations of felodipine and dehydrofelodipine were quantified by modification of a previously published method. ${ }^{37}$ Plasma samples were shipped under dry ice to the Department of Medicine, London Health Sciences Centre, London, Ontario, Canada, and stored at $-80^{\circ} \mathrm{C}$ until analysis. In brief, plasma $(500 \mu \mathrm{L})$ was extracted with toluene $(500 \mu \mathrm{L})$ containing the internal standard (H165/04; AB Haessle, Gotenburg, Sweden) by gentle oscillation of the mixture overnight. After centrifugation, a sample of the toluene extract $(1 \mu \mathrm{L})$ was introduced by splitless injection into a dual-tapered deactivated glass insert to prevent chemical oxidation of felodipine in the injector port. Chromatography was performed with a HewlettPackard 5890 Series II Gas Chromatograph equipped with a nickel 63 electron capture detector and a fused silica capillary column (internal diameter, $0.32 \mathrm{~mm} \times$ $25 \mathrm{~m}$ ) coated with a stationary phase of methyl silicone $(0.52 \mu \mathrm{m})$ (HP-1; Hewlett-Packard Canada Ltd, Mississauga, Ontario, Canada). After a purge for 1 minute, the initial oven temperature of $90^{\circ} \mathrm{C}$ was increased at $30^{\circ} \mathrm{C} / \mathrm{min}$ to $180^{\circ} \mathrm{C}$, at $5^{\circ} \mathrm{C} / \mathrm{min}$ to $260^{\circ} \mathrm{C}$ for 3 minutes, and then at $30^{\circ} \mathrm{C} / \mathrm{min}$ to a final temperature of $280^{\circ} \mathrm{C}$ for 5 minutes. The injector port and detector temperatures were maintained at $260^{\circ} \mathrm{C}$ and $300^{\circ} \mathrm{C}$, respectively. The carrier gas was ultrapure helium (column inlet pressure, $100 \mathrm{kPa}$ ), and the makeup gas was ultrapure nitrogen $(60 \mathrm{~mL} / \mathrm{min})$. The retention times of felodipine, dehydrofelodipine, and internal standard were 20.1, 14.5, and 21.7 minutes, respectively. The interday coefficients of variation for plasma felodipine and dehydrofelodipine concentration were $3.5 \%$ and 
$9.6 \%$, respectively, at $5 \mathrm{nmol} / \mathrm{L}$. The limit of quantitation was less than $0.5 \mathrm{nmol} / \mathrm{L}$ for each substance.

Plasma analysis for bergamottin and $6^{\prime}, 7^{\prime}$-dihydroxybergamottin. Plasma concentrations of bergamottin and $6^{\prime}, 7^{\prime}$-dihydroxybergamottin were quantified by LC-MS/MS. Plasma samples were shipped to the Department of Pharmacokinetics, Dynamics, and Metabolism, Pfizer Global Research and Development, Ann Arbor, Mich, and kept at $-80^{\circ} \mathrm{C}$ until analysis. Plasma samples obtained before dosing and at $0.5,1$, $1.5,2,2.5$, and 3 hours after dosing were analyzed, because initial experiments indicated no detectable bergamottin or $6^{\prime}, 7^{\prime}$-dihydroxybergamottin plasma concentrations at later times (4 to 24 hours).

Stock solutions of bergamottin, 6',7'-dihydroxybergamottin, and bergapten (internal standard) were prepared as $5 \mathrm{mg} / \mathrm{mL}$ in dimethylsulfoxide and then diluted to $10 \mu \mathrm{g} / \mathrm{mL}$ with mobile phase. Stock solutions were then serially diluted by use of blank plasma as necessary. The linear regression of the standard curves for bergamottin and 6',7'-dihydroxybergamottin showed $r^{2}=0.997$ and 0.999 , respectively, over the range of 1 to $200 \mathrm{ng} / \mathrm{mL}$.

For sample analysis, a $100-\mu \mathrm{L}$ aliquot of each plasma sample was mixed with $250 \mu \mathrm{L}$ of $50 \mathrm{ng} / \mathrm{mL}$ bergapten (internal standard) in acetonitrile and centrifuged at $4000 \mathrm{rpm}$ at $4{ }^{\circ} \mathrm{C}$ for 5 minutes. Supernatants were collected, evaporated to dryness, and reconstituted in $50 \mu \mathrm{L}$ of mobile phase $(50 \%$ acetonitrile/ $0.1 \%$ formic acid) for analysis. Aliquots $(5 \mu \mathrm{L})$ of plasma extracts were injected directly onto a MetaChem Polaris $\mathrm{C}_{18} 50 \times 2.0-\mathrm{mm}, 5-\mu \mathrm{m}$ column (ANSYS Technologies Inc, Torrance, Calif). Elution was performed with a step gradient of 50:50 ( $\mathrm{vol} / \mathrm{vol})$ acetonitrile/0.1\% formic acid (A) and 95:5 ( $\mathrm{vol} / \mathrm{vol}$ ) acetonitrile/0.1\% formic acid (B) at 4\% B initial conditions, with a step to $50 \% \mathrm{~B}$ at 1.0 minute and a step to $96 \% \mathrm{~B}$ at 1.2 minutes, and held before returning to initial conditions at 4 minutes. The column was maintained at room temperature, and the flow rate was $0.25 \mathrm{~mL} / \mathrm{min}$, with a total run time of 4 minutes. There was a 2-minute re-equilibrium period with the initial solvent mixture between analyses. Bergamottin, 6', 7'-dihydroxybergamottin, and bergapten eluted at approximately 2.6, 1.4, and 1.6 minutes, respectively.

Positive ion electrospray tandem mass spectra were recorded by use of an Applied Biosystems/MDSSCIEX model API 3000 triple-quadrupole mass spectrometer (Concord, Ontario, Canada) equipped with Applied Biosystems / MDS-SCIEX Analyst (version 1.2) operating software. The ion-spray voltage was set to $4500 \mathrm{~V}$, and the probe temperature was set at $450^{\circ} \mathrm{C}$.
Nitrogen was used as the collision gas, and the nebulizer, curtain, and collision gases were set to 6,10 , and 5 , respectively. Multiple reaction monitoring transitions of $m / z$ 339.1 $\rightarrow$ 203.1, $m / z 373.1 \rightarrow 203.2$, and $m / z 217.2 \rightarrow 202.1$ were used during quantitative analysis of bergamottin, 6', $7^{\prime}$-dihydroxybergamottin, and bergapten, respectively, with a dwell time of $300 \mathrm{~ms}$ for each transition.

Data analysis. One volunteer (subject 11) was found to have a measurable concentration of bergamottin in the predose plasma sample during the water control period, and all data from this individual were excluded from analysis for this violation in protocol. Plasma felodipine and dehydrofelodipine concentrations were analyzed by a noncompartmental method. The terminal elimination rate constant $\left(\mathrm{k}_{\mathrm{e}}\right)$ was determined by loglinear regression of the final data points (at least 3 ). The apparent elimination half-life $\left(\mathrm{t}_{1 / 2}\right)$ was calculated as $0.693 / \mathrm{k}_{\mathrm{e}}$. The area under the plasma concentration-time curve (AUC) was calculated by the linear trapezoidal method. Maximal plasma drug concentration $\left(\mathrm{C}_{\max }\right)$ and the time to reach $\mathrm{C}_{\max }\left(\mathrm{t}_{\max }\right)$ for felodipine, dehydrofelodipine, bergamottin, and $6^{\prime}, 7^{\prime}$-dihydroxybergamottin were obtained directly from the experimental data.

Statistical comparisons among control treatments and bergamottin treatments were initially done by 3-way ANOVA for repeated measures performed with SAS for Windows software (version 8.02; SAS Institute Inc, Cary, NC). The analysis model included sequence, subject nested within sequence, and drug formulation as factors. No sequence (ie, carryover) effects were observed for any pharmacokinetic parameter. For those ANOVA analyses of treatment effect with $P<$ .05 , post hoc a priori comparisons were performed between water and the 4 treatments with the paired $t$ test, with $P<.05$ being considered to indicate statistical significance. Results are presented as mean $\pm \mathrm{SD}$ in tables and mean \pm SEM in figures.

\section{RESULTS \\ Bergamottin concentration in grapefruit juice}

Grapefruit juice (Cedar Lite; Bromor Foods) contained $20.1 \pm 0.2 \mu \mathrm{mol} / \mathrm{L}$ bergamottin, or approximately $1.7 \mathrm{mg} / 250 \mathrm{~mL}$.

\section{Human pharmacokinetic study}

Effect of grapefruit juice and bergamottin. Grapefruit juice augmented plasma concentrations, $\mathrm{C}_{\max }$, and AUC of felodipine compared with water (Fig 2 and Table I). Felodipine $\mathrm{C}_{\max }$ and AUC from 0 to 12 hours $\left(\mathrm{AUC}_{0-12}\right)$ were increased by $89 \%$ (range, 

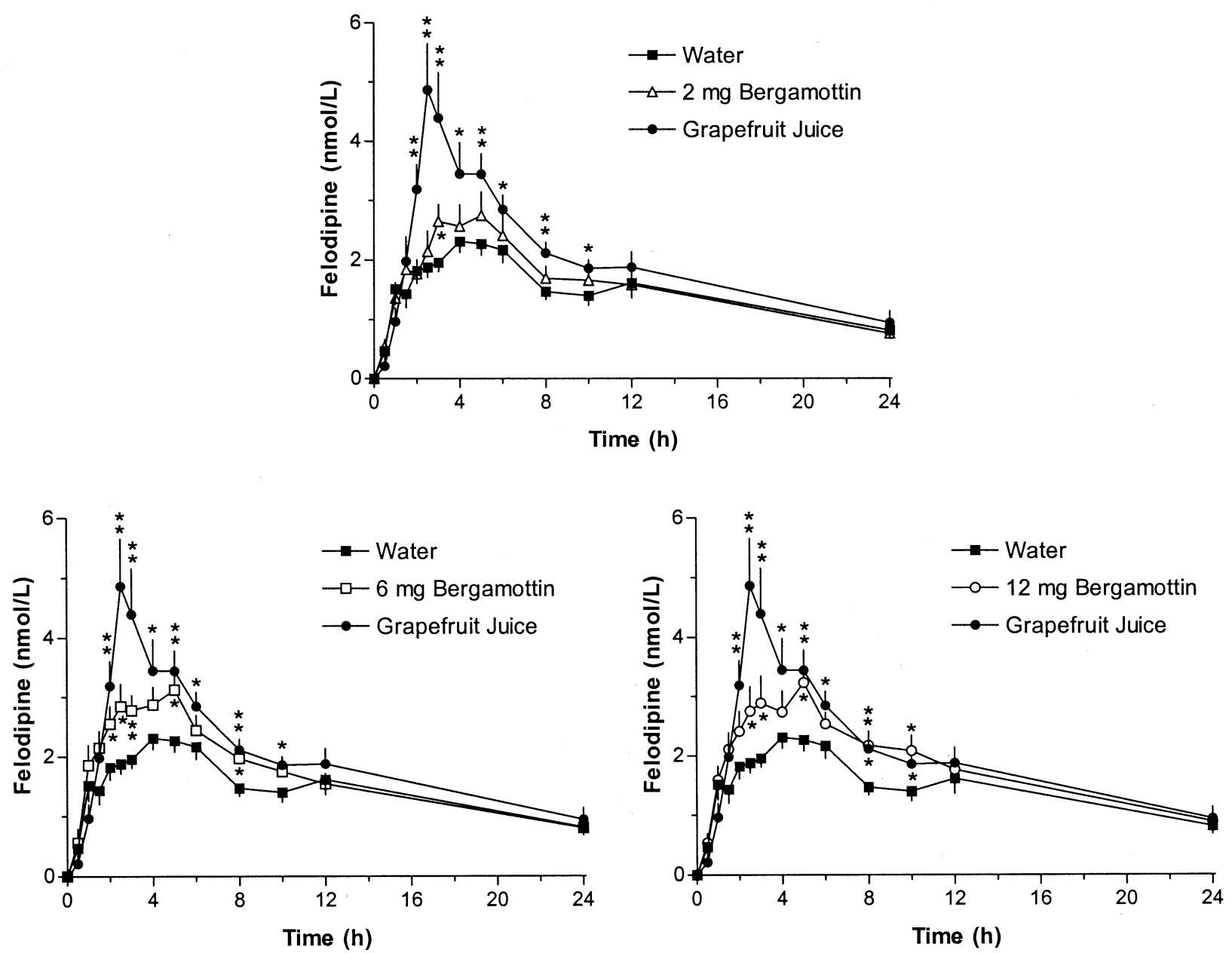

Fig 2. Mean plasma concentration-time profiles for felodipine with $250 \mathrm{~mL}$ grapefruit juice (solid circles); $2 \mathrm{mg}$ (open triangles), $6 \mathrm{mg}$ (open squares), or $12 \mathrm{mg}$ bergamottin (open circles); or 250 $\mathrm{mL}$ water (solid squares) in 11 healthy subjects. Error bars denote SE. Comparisons were made at each measurement time between the 4 treatments and water: 1 asterisk, $P<.05 ; 2$ asterisks, $P<.01$.

$-27 \%$ to $224 \% ; P<.025$ ) and $54 \%$ (range, $-24 \%$ to $139 \% ; P<.025$ ), respectively, compared with water. Plasma dehydrofelodipine concentrations, AUC, and $\mathrm{C}_{\max }$ values were increased. Dehydrofelodipine/felodipine AUC ratio (2.5 \pm 0.2 versus $2.1 \pm 0.2)$ and felodipine or dehydrofelodipine $t_{\max }$ or $t_{1 / 2}$ were not altered compared with those with water.

Bergamottin, $2 \mathrm{mg}$, increased felodipine $\mathrm{C}_{\max }$ by $33 \%$ (range, $-33 \%$ to $125 \% ; P<.05$ ) compared with water. Bergamottin, $6 \mathrm{mg}$, increased felodipine $\mathrm{C}_{\max }$ by $35 \%$ (range, $-30 \%$ to $86 \% ; P<.025$ ). Bergamottin, $12 \mathrm{mg}$, increased felodipine $\mathrm{C}_{\max }$ by $40 \%$ (range, $-9 \%$ to $120 \%$; $P<.05$ ) and $\mathrm{AUC}_{0-12}$ by $37 \%$ (range, $-8 \%$ to $124 \% ; P$ $<.05$ ) and dehydrofelodipine $\mathrm{AUC}_{0-12}$ by $41 \%$ (range, $-27 \% \pm 103 \% ; P<.025)$. Bergamottin administration did not affect dehydrofelodipine/felodipine AUC ratio or felodipine or dehydrofelodipine $t_{\max }$ or $t_{1 / 2}$.

The extent of the change in $\mathrm{C}_{\max }$ and change in AUC $_{0-12}$ of felodipine with grapefruit juice $(r=0.87$, $P<.001)$ or $2 \mathrm{mg}(r=0.75, P=.007), 6 \mathrm{mg}(r=$ $0.72, P=.012)$, or $12 \mathrm{mg}$ bergamottin $(r=0.77, P=$ .006) was highly correlated (Fig 3). However, a corresponding relationship was not observed between $t_{1 / 2}$ and AUC $_{0-12}$ of felodipine with grapefruit juice $(r=0.02$, $P=.96)$ or $2 \mathrm{mg}(r=0.04, P=.91), 6 \mathrm{mg}(r=0.19$, $P=.57)$, or $12 \mathrm{mg}$ bergamottin $(r=0.16, P=.64)$.

Plasma bergamottin and $6^{\prime}, 7^{\prime}$-dihydroxybergamottin concentrations. Data from the 8 subjects who had measurable plasma bergamottin levels are shown in Table II. One volunteer (subject 7) had a 
Table I. Pharmacokinetics of felodipine and its dehydrofelodipine metabolite after single dose administration of 5 -mg extended-release tablet of felodipine with $250 \mathrm{~mL}$ water; $2 \mathrm{mg}, 6 \mathrm{mg}$, or $12 \mathrm{mg}$ bergamottin; or grapefruit juice $(\mathrm{N}=11)$

\begin{tabular}{|c|c|c|c|c|c|}
\hline & \multirow[b]{2}{*}{ Water } & \multicolumn{3}{|c|}{ Bergamottin } & \multirow{2}{*}{$\begin{array}{l}\text { Grapefruit } \\
\quad \text { juice }\end{array}$} \\
\hline & & $2 \mathrm{mg}$ & $6 \mathrm{mg}$ & $12 \mathrm{mg}$ & \\
\hline \multicolumn{6}{|l|}{ Felodipine } \\
\hline $\mathrm{AUC}_{\mathrm{O}-12}(\mathrm{nmol} / \mathrm{L} \cdot \mathrm{h})$ & $20.1 \pm 4.4$ & $22.8 \pm 6.8$ & $25.6 \pm 7.3$ & $26.7 \pm 8.3^{*}$ & $29.8 \pm 7.8 * *$ \\
\hline $\mathrm{AUC}_{0-24}(\mathrm{nmol} / \mathrm{L} \cdot \mathrm{h})$ & $34.1 \pm 10.2$ & $36.1 \pm 10.5$ & $39.0 \pm 11.2$ & $42.0 \pm 12.9$ & $46.0 \pm 12.8^{* *}$ \\
\hline $\mathrm{C}_{\max }(\mathrm{nmol} / \mathrm{L})$ & $2.8 \pm 0.5$ & $3.6 \pm 1.0^{*}$ & $3.7 \pm 1.0 * *$ & $3.9 \pm 1.3^{*}$ & $5.2 \pm 2.3 * *$ \\
\hline$t_{\max }(h)$ & $5.0 \pm 3.9$ & $5.1 \pm 1.9$ & $3.7 \pm 1.2$ & $4.5 \pm 2.2$ & $3.0 \pm 1.4$ \\
\hline$t_{1 / 2}(h)$ & $22.9 \pm 24.0$ & $13.0 \pm 9.9$ & $12.9 \pm 6.6$ & $13.0 \pm 6.2$ & $12.8 \pm 9.5$ \\
\hline \multicolumn{6}{|l|}{ Dehydrofelodipine } \\
\hline $\mathrm{AUC}_{\mathrm{O}-12}(\mathrm{nmol} / \mathrm{L} \cdot \mathrm{h})$ & $47.9 \pm 11.8$ & $53.3 \pm 20.1$ & $61.7 \pm 23.4$ & $63.1 \pm 13.3^{* *}$ & $60.8 \pm 23.6^{* *}$ \\
\hline $\mathrm{AUC}_{0-24}(\mathrm{nmol} / \mathrm{L} \cdot \mathrm{h})$ & $68.7 \pm 23.5$ & $69.3 \pm 23.0$ & $86.3 \pm 37.3$ & $83.8 \pm 15.5$ & $77.2 \pm 33.1$ \\
\hline $\mathrm{C}_{\max }(\mathrm{nmol} / \mathrm{L})$ & $9.3 \pm 3.7$ & $9.4 \pm 3.2$ & $10.6 \pm 2.7$ & $10.3 \pm 2.6$ & $12.4 \pm 3.6^{*}$ \\
\hline $\mathrm{t}_{\max }(\mathrm{h})$ & $5.1 \pm 4.2$ & $4.5 \pm 2.2$ & $3.1 \pm 1.3$ & $3.2 \pm 1.4$ & $3.3 \pm 1.8$ \\
\hline$t_{1 / 2}(h)$ & $7.5 \pm 6.2$ & $5.6 \pm 1.5$ & $6.3 \pm 4.3$ & $9.4 \pm 10.9$ & $4.8 \pm 0.9$ \\
\hline \multicolumn{6}{|c|}{ Dehydrofelodipine/felodipine } \\
\hline $\mathrm{AUC}_{0-12}(\mathrm{nmol} / \mathrm{L} \cdot \mathrm{h})$ & $2.5 \pm 0.7$ & $2.3 \pm 0.6$ & $2.4 \pm 0.8$ & $2.4 \pm 0.4$ & $2.1 \pm 0.7$ \\
\hline
\end{tabular}

single quantifiable bergamottin concentration at 0.5 hour after administration of grapefruit juice or $2 \mathrm{mg}$ bergamottin. Six and eight volunteers had at least 1 quantifiable concentration and $t_{\max }$ of bergamottin ranging between 0.5 and 2.5 hours after ingestion of 6 $\mathrm{mg}$ and $12 \mathrm{mg}$ bergamottin, respectively. The plasma levels of bergamottin after a 12-mg dose were variable and significantly higher in 1 subject. Mean $\mathrm{C}_{\max }$ values of bergamottin among individuals after $6 \mathrm{mg}$ bergamottin tended to be lower than those after $12 \mathrm{mg}$ bergamottin. Two volunteers (subjects 6 and 7) had 4 detectable plasma concentrations of $6^{\prime}, 7^{\prime}$-dihydroxybergamottin that were below the limit of quantification (1 ng/mL). One volunteer (subject 8) had quantifiable plasma levels of $6^{\prime}, 7^{\prime}$-dihydroxybergamottin as shown in Table II.

Adverse events. All treatments were generally well tolerated. Adverse events were mild and resolved during the study day without medical intervention. They included headache, dizziness, fatigue, muscle cramps, and nausea. The number of events in each treatment group was as follows: control, 8; grapefruit juice, 19; 2 $\mathrm{mg}$ bergamottin, 12; $6 \mathrm{mg}$ bergamottin, 8; and $12 \mathrm{mg}$ bergamottin, 12 .

\section{DISCUSSION}

To our knowledge, this is the first report to evaluate the effect of bergamottin as an active ingredient in grapefruit juice-drug interactions by administering it as a pure substance. Furanocoumarins have been known for some time to inhibit in vitro oxidative metabolism mediated by CYP3A $4^{22,25}$ and are considered to be the active ingredients responsible for the grapefruit juice effect. ${ }^{15}$ However, previous clinical investigations assessing the role of furanocoumarins have administered impure fractions from grapefruit juice or tested the effect of the citrus juices, lime juice, or Seville (bitter) orange juice, which also contain certain furanocoumarins found in grapefruit juice. ${ }^{15,29,30,33,38,39}$ An alternative, likely more desirable approach that was taken in this study was to test the clinical effect of a potentially important furanocoumarin by administering it as a pure substance to humans.

In this study a single oral dose of $2 \mathrm{mg}$ bergamottin, approximating the amount in a normal-sized glass of grapefruit juice $(1.7 \mathrm{mg})$, did augment the $\mathrm{C}_{\max }$ of felodipine. Although the AUC of felodipine was not statistically increased, the high correlation between the change in $\mathrm{C}_{\max }$ and AUC for felodipine among individuals indicated that the oral bioavailability of felodipine might be increased by $2 \mathrm{mg}$ bergamottin. However, this was not apparent, most likely because the effect was markedly less than that observed with whole grapefruit juice. These results are in agreement with 


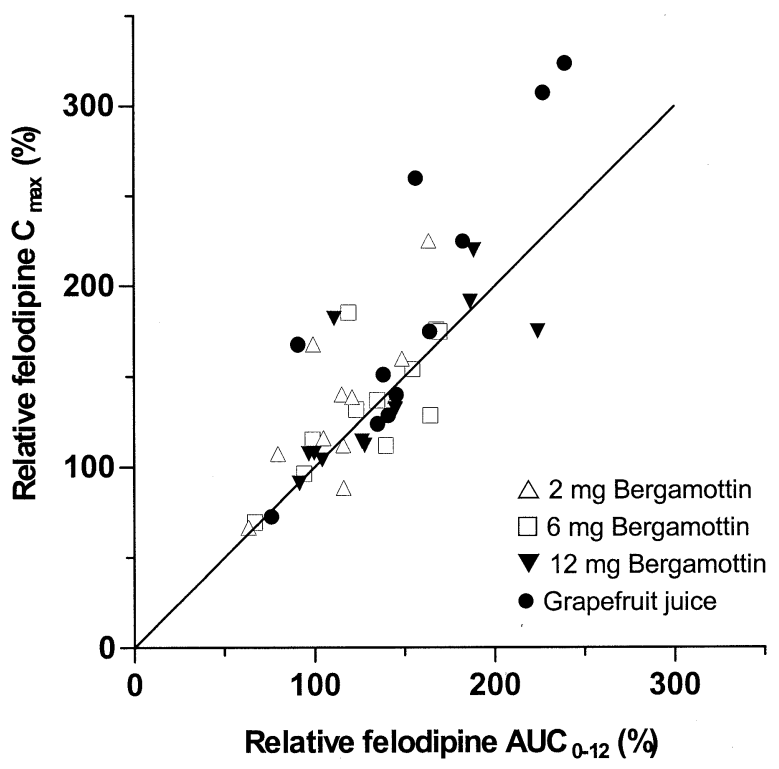

Fig 3. Relative felodipine maximal plasma drug concentration $\left(\mathrm{C}_{\max }\right)$ (bergamottin or grapefruit juice relative to water expressed as percent) plotted against relative felodipine area under plasma concentration-time curve from 0 to 12 hours $\left(\mathrm{AUC}_{0-12}\right)$ for each individual with $250 \mathrm{~mL}$ grapefruit juice (circles) or $2 \mathrm{mg}$ (open triangles), $6 \mathrm{mg}$ (squares), or $12 \mathrm{mg}$ bergamottin (solid triangles). The diagonal line represents the line of unity.

those obtained after administration of lime juice or grapefruit juice containing comparable amounts of bergamottin, ${ }^{33}$ supporting a contribution of bergamottin to the grapefruit juice interaction with felodipine.

In this study $6 \mathrm{mg}$ and $12 \mathrm{mg}$ bergamottin had an effect that was not substantially greater than that of 2 $\mathrm{mg}$ bergamottin and less than that of grapefruit juice. With the assumption that bergamottin given as a pure substance has the same disposition as bergamottin in whole grapefruit juice, it would also appear that bergamottin is not the only active ingredient and that additional substances contribute to the magnitude of the interaction. Given that $2 \mathrm{mg}$ bergamottin accounted for only a mean $30 \%$ of the grapefruit juice effect, these additional ingredients might be expected to contribute substantially to the interaction.

In a previous study grapefruit juice was separated by high-speed centrifugation and ultrafiltration into a particulate fraction, which contained all of the bergamottin, and a supernatant fraction, which contained most of the $6^{\prime}, 7^{\prime}$-dihydroxybergamottin. ${ }^{29}$ Results with the particulate fraction indicated that bergamottin might be a major active constituent. ${ }^{21,29}$ However, it should be noted the particulate material also contains trace amounts of at least 3 other furanocoumarins that are dimer derivatives of $6^{\prime}, 7^{\prime}$-dihydroxybergamottin and potent in vitro inhibitors of CYP3A4..$^{21,23,40}$ Thus these other furanocoumarins may contribute substantially to the clinical interaction between grapefruit juice and drugs. ${ }^{21}$ Grapefruit juice coadministration with felodipine generally results in 1.5- to 3.5-fold increases in felodipine AUC. ${ }^{3}$ In this study grapefruit juice increases in mean felodipine AUC (1.5-fold) and $\mathrm{C}_{\max }$ (1.9-fold) were modest even though bergamottin concentrations in the juice were greater than average, ${ }^{12,20,21}$ implicating a contribution of other active components and lower concentrations of active ingredients present in the brand of juice studied. Moreover, the in vitro observation that the inhibitory effect of furanocoumarins on the activity of CYP3A4 only approximates that of grapefruit juice when a combination of several furanocoumarins are simultaneously tested at highest concentrations suggested that the mechanism might involve an additive or synergistic interaction among active furanocoumarins. ${ }^{12,21,23,40,41}$ Therefore it would be of interest to investigate the effect of a cocktail of furanocoumarins at concentrations similar to those present in grapefruit juice on in vivo drug disposition.

Although $2 \mathrm{mg}$ bergamottin produced a modest mean increase in the oral bioavailability of felodipine, the magnitude of the interaction was highly variable among individuals. Certain individuals had more than a doubling in the plasma concentration of felodipine. Moreover, the magnitude of the effect was consistent within individuals with repeat administration of bergamottin. Thus the presence of bergamottin in fruit juices and possibly other food sources or herbal supplements might produce clinically significant drug interactions in certain susceptible individuals.

This variability of effect among individuals might be explained by variable bioavailability or altered disposition of bergamottin, possibly from low or inconsistent permeability into cells containing CYP3A4. ${ }^{42}$ However, the measurement of bergamottin in plasma samples, particularly at the higher doses, indicates that this substance was likely accessible to intestinal and hepatic CYP3A4. Because felodipine is not a substrate of P-glycoprotein, it is unlikely that the previously reported in vitro inhibitory activity of bergamottin toward this efflux transporter could explain the inconsistent effect among subjects. ${ }^{31,43}$ Previously, it was demonstrated that individuals with the highest baseline enterocyte content of CYP3A4 had the largest increase in felodipine bioavailability with grapefruit juice. ${ }^{8}$ Thus 
Table II. Individual pharmacokinetics $(\mathrm{n}=8)$ of bergamottin and $6^{\prime}, 7^{\prime}$-dihydroxybergamottin after administration of grapefruit juice or $2 \mathrm{mg}, 6 \mathrm{mg}$, or $12 \mathrm{mg}$ bergamottin

\begin{tabular}{|c|c|c|c|c|c|c|c|c|c|c|c|c|}
\hline \multirow{3}{*}{$\begin{array}{l}\text { Subject } \\
\text { No. }\end{array}$} & \multicolumn{4}{|c|}{$\begin{array}{l}\text { No. of samples containing } B G^{*} \text { or } \\
\qquad \mathrm{DB}^{\dagger}\end{array}$} & \multicolumn{4}{|c|}{$C_{\max }(n g / m L)$} & \multicolumn{4}{|c|}{$t_{\max }(h)$} \\
\hline & \multirow[b]{2}{*}{$G F J$} & \multicolumn{3}{|c|}{$B G$} & \multirow[b]{2}{*}{$G F J$} & \multicolumn{3}{|c|}{$B G$} & \multirow[b]{2}{*}{$G F J$} & \multicolumn{3}{|c|}{$B G$} \\
\hline & & $2 \mathrm{mg}$ & $6 \mathrm{mg}$ & $12 \mathrm{mg}$ & & $2 \mathrm{mg}$ & $6 \mathrm{mg}$ & $12 \mathrm{mg}$ & & $2 \mathrm{mg}$ & $6 \mathrm{mg}$ & $12 \mathrm{mg}$ \\
\hline 1 & - & - & 3 & 3 & - & - & 1.9 & 4.9 & - & - & 1.0 & 1.0 \\
\hline 3 & - & - & 1 & 1 & - & - & 1.6 & 2.4 & - & - & 0.5 & 0.5 \\
\hline 4 & - & - & 3 & 4 & - & - & 2.1 & 2.8 & - & - & 0.5 & 1.0 \\
\hline 5 & - & - & - & 3 & - & - & - & 2.9 & - & - & - & 1.0 \\
\hline 6 & - & - & - & 4 & - & - & - & 6.1 & - & - & - & 1.0 \\
\hline 7 & 1 & 1 & 2 & 3 & 1.2 & 6.7 & 2.5 & 2.8 & 0.5 & 0.5 & 1.0 & 1.5 \\
\hline 8 & - & - & 1 & 2 & - & - & 1.7 & 23 & - & - & 0.5 & 0.5 \\
\hline $8 \dagger$ & - & - & - & - & 3 & - & - & - & 5.9 & - & - & 0.5 \\
\hline 9 & - & - & 2 & 4 & - & - & 2.7 & 2.4 & - & - & 1.0 & 2.5 \\
\hline Mean & & & & & & & $2.1 \S$ & $5.9 \|$ & & & $0.8 \S$ & $1.1 \|$ \\
\hline SD & & & & & & & 0.4 & 6.9 & & & 0.3 & 0.6 \\
\hline
\end{tabular}

BG, Bergamottin; DHB, 6', $7^{\prime}$-dihydroxybergamottin; GFJ, grapefruit juice.

*Number of samples in which bergamottin was detected (ie, $>1 \mathrm{ng} / \mathrm{mL}$ ) (samples analyzed from 0 to 3 hours).

$\uparrow$ Number of samples in which $6^{\prime}, 7^{\prime}$-dihydroxybergamottin was detected (ie, $>1 \mathrm{ng} / \mathrm{mL}$ ) (samples analyzed from 0 to 3 hours).

$\$$ Mean and SD values are presented for bergamottin plasma levels.

$\S \mathrm{n}=6$.

$\| \mathrm{n}=8$.

the most viable cause for the variability in the extent of the interaction with felodipine among individuals appears to be inherent variation in the enterocyte content of CYP3A4.

$6^{\prime}, 7^{\prime}$-Dihydroxybergamottin was quantified in the plasma of 1 subject, and the peak concentration of this metabolite was rapid and occurred at the same time as that of the parent compound. Thus biotransformation of bergamottin to the dihydroxylated product appears to occur in vivo and to be rapid. Furanocoumarins are believed to act clinically by mechanism-based inactivation of CYP3A4. The plasma measurement of $6^{\prime}, 7^{\prime}-$ dihydroxybergamottin suggested that the reactive substance might be a chemically unstable intermediate of these 2 substances, in addition to the proposed bioactivation of the furan ring. ${ }^{44}$ Moreover, in vitro irreversible kinetic parameters for bergamottin indicate that an in vivo drug interaction with CYP3A4 substrates is either "likely" or "possible." "22,23,26,45 However, current predictive models for clinical drug interactions with irreversible enzyme inhibitors are still being developed and do not account for an interaction at the level of the enterocyte. ${ }^{46}$

Biotransformation of felodipine by CYP3A4 normally yields a single inactive metabolite, dehydrofelodipine. Previous studies show that grapefruit juice increased the AUC of felodipine and decreased the dehydrofelodipine/felodipine AUC ratio, suggesting inhibition of the primary metabolic pathway. ${ }^{4}$ In this study neither grapefruit juice nor bergamottin decreased the dehydrofelodipine/felodipine AUC ratio to a statistically significant extent, possibly as a result of the insufficient magnitude of the increase in the AUC of felodipine as a result of the study population and the batch of grapefruit juice tested.

In summary, bergamottin administered as a pure substance enhanced the oral bioavailability of felodipine. However, the effect was substantially less than that produced by grapefruit juice even at markedly higher doses of bergamottin than normally present in the juice. Nevertheless, foods containing only bergamottin would likely cause a clinically relevant drug interaction in susceptible individuals. It appears probable that the interaction also involves other furanocoumarins present in whole grapefruit juice, possibly acting in combination by additive or synergistic mechanisms. Bergamottin has systemic availability and is metabolized to $6^{\prime}, 7^{\prime}$ dihydroxybergamottin in humans.

We acknowledge Dr Faans Steyn for statistical analysis of data. Pfizer Inc has a patent (US 6,509,371) on compositions containing bergamottin for increasing the oral bioavailability of pharmaceutical agents, coinvented by Drs He and Hollenberg. Drs Goosen, Yu, Cohen, and Williams are employees of and have stock in Pfizer Inc. Dr He was an employee of Pfizer Inc and is currently employed by 
Bristol-Myers Squibb. Dr Hollenberg has consulted for, received research funding from, and has stock in Pfizer Inc. Dr Woster is a coinventor of a patent on the synthesis and use of $6^{\prime}, 7^{\prime}$ dihydroxybergamottin (US 6,160,006). Ms Cillié and Drs Bailey, Rheeders, and Dijkstra have no conflict of interest.

\section{References}

1. Bailey DG, Spence JD, Munoz C, Arnold JM. Interaction of citrus juices with felodipine and nifedipine. Lancet 1991;337:268-9.

2. Edgar B, Regardh CG, Johnsson G, Johansson L, Lundborg P, Lofberg I, et al. Felodipine kinetics in healthy men. Clin Pharmacol Ther 1985;38:205-11.

3. Bailey DG, Arnold JM, Spence JD. Grapefruit juice-drug interactions. Br J Clin Pharmacol 1998;46:101-10.

4. Bailey DG, Bend JR, Arnold JM, Tran LT, Spence JD. Erythromycin-felodipine interaction: magnitude, mechanism, and comparison with grapefruit juice. Clin Pharmacol Ther 1996;60:25-33.

5. Ducharme MP, Warbasse LH, Edwards DJ. Disposition of intravenous and oral cyclosporine after administration with grapefruit juice. Clin Pharmacol Ther 1995;57:48591.

6. Kupferschmidt HH, Ha HR, Ziegler WH, Meier PJ, Krahenbuhl S. Interaction between grapefruit juice and midazolam in humans. Clin Pharmacol Ther 1995;58:20-8.

7. Lundahl J, Regardh CG, Edgar B, Johnsson G. Effects of grapefruit juice ingestion-pharmacokinetics and haemodynamics of intravenously and orally administered felodipine in healthy men. Eur J Clin Pharmacol 1997; 52:139-45.

8. Lown KS, Bailey DG, Fontana RJ, Janardan SK, Adair $\mathrm{CH}$, Fortlage LA, et al. Grapefruit juice increases felodipine oral availability in humans by decreasing intestinal CYP3A protein expression. J Clin Invest 1997;99:254553.

9. Correia MA, Davoll SH, Wrighton SA, Thomas PE. Degradation of rat liver cytochromes P450 3A after their inactivation by 3,5-dicarbethoxy-2,6-dimethyl-4-ethyl1,4-dihydropyridine: characterization of the proteolytic system. Arch Biochem Biophys 1992;297:228-38.

10. Takanaga H, Ohnishi A, Matsuo H, Sawada Y. Inhibition of vinblastine efflux mediated by P-glycoprotein by grapefruit juice components in Caco-2 cells. Biol Pharm Bull 1998;21:1062-6.

11. Eagling VA, Profit L, Back DJ. Inhibition of the CYP3A4-mediated metabolism and P-glycoproteinmediated transport of the HIV-1 protease inhibitor saquinavir by grapefruit juice components. Br J Clin Pharmacol 1999;48:543-52.

12. Ohnishi A, Matsuo H, Yamada S, Takanaga H, Morimoto $\mathrm{S}$, Shoyama Y, et al. Effect of furanocoumarin derivatives in grapefruit juice on the uptake of vinblastine by Caco-2 cells and on the activity of cytochrome P450 3A4. Br J Pharmacol 2000;130:1369-77.
13. Spahn-Langguth H, Langguth P. Grapefruit juice enhances intestinal absorption of the P-glycoprotein substrate talinolol. Eur J Pharm Sci 2001;12:361-7.

14. Becquemont L, Verstuyft C, Kerb R, Brinkmann U, Lebot M, Jaillon $\mathrm{P}$, et al. Effect of grapefruit juice on digoxin pharmacokinetics in humans. Clin Pharmacol Ther 2001;70:311-6.

15. Malhotra S, Bailey DG, Paine MF, Watkins PB. Seville orange juice-felodipine interaction: comparison with dilute grapefruit juice and involvement of furocoumarins. Clin Pharmacol Ther 2001;69:14-23.

16. Dresser GK, Bailey DG, Carruthers SG. Grapefruit juicefelodipine interaction in the elderly. Clin Pharmacol Ther 2000;68:28-34.

17. Bailey DG, Arnold JM, Strong HA, Munoz C, Spence JD. Effect of grapefruit juice and naringin on nisoldipine pharmacokinetics. Clin Pharmacol Ther 1993;54:589-94.

18. Bailey DG, Arnold JM, Munoz C, Spence JD. Grapefruit juice-felodipine interaction: mechanism, predictability, and effect of naringin. Clin Pharmacol Ther 1993;53: 637-42.

19. Rashid T, McKinstry C, Renwick AG, Dirnhuber M, Waller DG, George CF. Quercetin, an in vitro inhibitor of CYP3A, does not contribute to the interaction between nifedipine and grapefruit juice. $\mathrm{Br} \mathrm{J}$ Clin Pharmacol 1993;36:460-3.

20. Fukuda K, Guo L, Ohashi N, Yoshikawa M, Yamazoe Y. Amounts and variation in grapefruit juice of the main components causing grapefruit-drug interaction. J Chromatogr B Biomed Sci Appl 2000;741:195-203.

21. Guo LQ, Fukuda K, Ohta T, Yamazoe Y. Role of furanocoumarin derivatives on grapefruit juice-mediated inhibition of human CYP3A activity. Drug Metab Dispos 2000;28:766-71.

22. He K, Iyer KR, Hayes RN, Sinz MW, Woolf TF, Hollenberg PF. Inactivation of cytochrome P450 3A4 by bergamottin, a component of grapefruit juice. Chem Res Toxicol 1998;11:252-9.

23. Tassaneeyakul W, Guo LQ, Fukuda K, Ohta T, Yamazoe Y. Inhibition selectivity of grapefruit juice components on human cytochromes P450. Arch Biochem Biophys 2000;378:356-63.

24. Ohta T, Nagahashi M, Hosoi S, Tsukamoto S. Dihydroxybergamottin caproate as a potent and stable CYP3A4 inhibitor. Bioorg Med Chem 2002;10:969-73.

25. Schmiedlin-Ren P, Edwards DJ, Fitzsimmons ME, He K, Lown KS, Woster PM, et al. Mechanisms of enhanced oral availability of CYP3A4 substrates by grapefruit constituents. Decreased enterocyte CYP3A4 concentration and mechanism-based inactivation by furanocoumarins. Drug Metab Dispos 1997;25:1228-33.

26. Greenblatt DJ, von Moltke LL, Harmatz JS, Chen G, Weemhoff JL, Jen C, et al. Time course of recovery of cytochrome P450 3A function after single doses of grapefruit juice. Clin Pharmacol Ther 2003;74:121-9. 
27. Edwards DJ, Bellevue FH III, Woster PM. Identification of 6',7'-dihydroxybergamottin, a cytochrome P450 inhibitor, in grapefruit juice. Drug Metab Dispos 1996;24: 1287-90.

28. Guo LQ, Taniguchi M, Xiao YQ, Baba K, Ohta T, Yamazoe Y. Inhibitory effect of natural furanocoumarins on human microsomal cytochrome P450 3A activity. Jpn J Pharmacol 2000;82:122-9.

29. Bailey DG, Kreeft JH, Munoz C, Freeman DJ, Bend JR. Grapefruit juice-felodipine interaction: effect of naringin and 6',7'-dihydroxybergamottin in humans. Clin Pharmacol Ther 1998;64:248-56.

30. Kakar SM, Paine MF, Stewart PW, Watkins PB. 6' $7^{\prime}$ Dihydroxybergamottin contributes to the grapefruit juice effect. Clin Pharmacol Ther 2004;75:569-79.

31. Wang EJ, Casciano CN, Clement RP, Johnson WW. Inhibition of P-glycoprotein transport function by grapefruit juice psoralen. Pharm Res 2001;18:432-8.

32. Fukuda K, Ohta T, Oshima Y, Ohashi N, Yoshikawa M, Yamazoe Y. Specific CYP3A4 inhibitors in grapefruit juice: furocoumarin dimers as components of drug interaction. Pharmacogenetics 1997;7:391-6.

33. Bailey DG, Dresser GK, Bend JR. Bergamottin, lime juice, and red wine as inhibitors of cytochrome P450 3A4 activity: comparison with grapefruit juice. Clin Pharmacol Ther 2003;73:529-37.

34. Sahi J, Reyner EL, Bauman JN, Gueneva-Boucheva K, Burleigh JE, Thomas VH. The effect of bergamottin on diazepam plasma levels and P450 enzymes in beagle dogs. Drug Metab Dispos 2002;30:135-40.

35. Goosen TC, Van Rooyen J, Erasmus E, Potgieter HC. Cyclosporine pharmacokinetics following co-administration with grapefruit juice, bergamottin or vitamin E TPGS in rats [abstract]. Pharmacologist 2002;44:A172.

36. Mohri K, Uesawa Y. Effects of furanocoumarin derivatives in grapefruit juice on nifedipine pharmacokinetics in rats. Pharm Res 2001;18:177-82.
37. Ahnoff M. Determination of felodipine in plasma by capillary gas chromatography with electron capture detection. J Pharm Biomed Anal 1984;2:519-26.

38. Bailey DG, Dresser GK, Kreeft JH, Munoz C, Freeman DJ, Bend JR. Grapefruit-felodipine interaction: effect of unprocessed fruit and probable active ingredients. Clin Pharmacol Ther 2000;68:468-77.

39. Paine MF, Widmer W, Hart HL, Brown SS, Thomas BF, Pusek SN, et al. A furanocoumarin-free grapefruit juice does not alter the oral pharmacokinetics of felodipine [abstract]. Drug Metab Rev 2003;35(Suppl 2):67.

40. Ohta T, Maruyama T, Nagahashi M, Miyamoto Y, Hosoi S, Kiuchi F, et al. Paradisin C: a new CYP3A4 inhibitor from grapefruit juice. Tetrahedron 2002;58:6631-5.

41. Ho PC, Saville DJ, Wanwimolruk S. Inhibition of human CYP3A4 activity by grapefruit flavonoids, furanocoumarins and related compounds. J Pharm Pharm Sci 2001;4: 217-27.

42. Criss AB, Paine MF, Watkins PB. The two major furocoumarins in grapefruit juice have distinct absorption and protein binding characteristics [abstract]. Drug Metab Rev 2002;34(Suppl 1):169.

43. Cummins CL, Jacobsen W, Benet LZ. Unmasking the dynamic interplay between intestinal P-glycoprotein and CYP3A4. J Pharmacol Exp Ther 2002;300:1036-45.

44. Koenigs LL, Trager WF. Mechanism-based inactivation of cytochrome P450 2B1 by 8-methoxypsoralen and several other furanocoumarins. Biochemistry 1998;37: 13184-93.

45. Wrighton SA, Schuetz EG, Thummel KE, Shen DD, Korzekwa KR, Watkins PB. The human CYP3A subfamily: practical considerations. Drug Metab Rev 2000;32: 339-61.

46. Mayhew BS, Jones DR, Hall SD. An in vitro model for predicting in vivo inhibition of cytochrome P450 3A4 by metabolic intermediate complex formation. Drug Metab Dispos 2000;28:1031-7. 